\title{
Pre-diagnosis plasma immune markers and risk of non-Hodgkin lymphoma in two prospective cohort studies
}

Mara M. Epstein ${ }^{1}$ Bernard Rosner, ${ }^{2,3}$ Elizabeth C. Breen ${ }^{4,5}$ Julie L. Batista ${ }^{3}$ Edward L. Giovannucci, ${ }^{3,6,7}$ Larry Magpantay, ${ }^{4,8}$ Jon C. Aster, ${ }^{9}$ Scott J. Rodig, ${ }^{9}$ Kimberly A. Bertrand, ${ }^{10}$ Francine Laden, ${ }^{3,7}$ Otoniel Martínez-Maza ${ }^{4,8,11,12,13}$ and Brenda M. Birmann ${ }^{3}$

${ }^{1}$ Department of Medicine and the Meyers Primary Care Institute, University of Massachusetts Medical School, Worcester, MA; ${ }^{2}$ Department of Biostatistics, Harvard T.H. Chan School of Public Health, Boston, MA; ${ }^{3}$ Channing Division of Network Medicine, Department of Medicine, Brigham and Women's Hospital and Harvard Medical School, Boston, MA; ${ }^{4}$ UCLA AIDS Institute, Los Angeles, CA; ${ }^{5}$ Department of Psychiatry \& Biobehavioral Sciences, David Geffen School of Medicine at UCLA, Los Angeles, CA; ${ }^{6}$ Department of Nutrition, Harvard T.H. Chan School of Public Health, Boston, MA; ${ }^{7}$ Department of Epidemiology, Harvard T.H. Chan School of Public Health, Boston, MA; ${ }^{8}$ Department of Obstetrics \& Gynecology, David Geffen School of Medicine at UCLA, Los Angeles, CA; 'Department of Pathology, Brigham and Women's Hospital and Harvard Medical School, Boston, MA; ${ }^{10}$ Slone Epidemiology Center at Boston University, Boston, MA; ${ }^{11}$ Department of Epidemiology, UCLA Fielding School of Public Health, Los Angeles, CA; ${ }^{12}$ Department of Microbiology, Immunology \& Molecular Genetics, David Geffen School of Medicine at UCLA, Los Angeles, CA and ${ }^{13}$ Jonsson Comprehensive Cancer Center, David Geffen School of Medicine at UCLA, Los Angeles, CA, USA

\section{ABSTRACT}

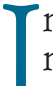
nflammation and B-cell hyperactivation have been associated with non-Hodgkin lymphoma development. This prospective analysis aimed to further elucidate pre-diagnosis plasma immune marker profiles associated with non-Hodgkin lymphoma risk. We identified 598 incident lymphoma cases and 601 matched controls in Nurses' Health Study and Health Professionals Follow-up Study participants with archived pre-diagnosis plasma samples and measured 13 immune marker levels with multiplexed immunoassays. Using multivariable logistic regression we calculated Odds Ratios (OR) and 95\% Confidence Intervals (CI) per standard deviation unit increase in biomarker concentration for risk of non-Hodgkin lymphoma and major histological subtype, stratifying additional models by years $(<5,5$ to $<10, \geq 10)$ after blood draw. Soluble interleukin-2 receptor- $\alpha$, CXC chemokine ligand 13 , soluble CD30, and soluble tumor necrosis factor receptor-2 were individually positively associated, and B-cell activating factor of the tumor necrosis factor family inversely associated, with all non-Hodgkin lymphoma and one or more subtypes. The biomarker combinations associated independently with lymphoma varied somewhat by subtype and years after blood draw. Of note, the unexpected inverse association between B-cell activating factor and chronic lymphocytic leukemia/small lymphocytic lymphoma risk (OR: 95\%CI: 0.51, 0.43-0.62) persisted more than ten years after blood draw (OR: 0.70; 95\% CI: 0.52-0.93). In conclusion, immune activation precedes non-Hodgkin lymphoma diagnosis by several years. Decreased B-cell activating factor levels may denote nascent chronic lymphocytic leukemia many years pre-diagnosis.

\section{Introduction}

Severe immune compromise is a strong risk factor for non-Hodgkin lymphoma (NHL), and B-cell activation and inflammation have been associated with an increased risk of AIDS-related NHL. Elevated pre-diagnosis plasma levels of markers of B-cell stimulation including CXC chemokine ligand 13 (CXCL13; a B-cell attracting chemokine), ${ }^{1}$ interleukin (IL)-6 (a B-cell stimulatory cytokine), and soluble (s) CD30 (sCD30; a soluble receptor indicative of $\mathrm{B}$ - and T-cell activation) pre-
Haematologica 2018

Volume 103(10):1679-1687

\section{Correspondence:}

brenda.birmann@channing.havard.edu

Received: November 7, 2017.

Accepted: June 15, 2018.

Pre-published: June 21, 2018.

doi:10.3324/haematol.2017.183236

Check the online version for the most updated information on this article, online supplements, and information on authorship \& disclosures: www.haematologica.org/content/103/10/1679

(C)2018 Ferrata Storti Foundation

Material published in Haematologica is covered by copyright. All rights are reserved to the Ferrata Storti Foundation. Use of published material is allowed under the following terms and conditions:

https://creativecommons.org/licenses/by-nc/4.0/legalcode. Copies of published material are allowed for personal or internal use. Sharing published material for non-commercial purposes is subject to the following conditions:

https://creativecommons.org//licenses/by-nc/4.0/legalcode, sect. 3. Reproducing and sharing published material for commercial purposes is not allowed without permission in writing from the publisher. 
dicted risk of an AIDS-NHL diagnosis in HIV-positive persons, ${ }^{2.4}$ in some instances as early as five years pre-diagnosis. ${ }^{5}$ Several of these markers have also demonstrated an association with NHL risk in immunocompetent people in prospective studies. ${ }^{6-13}$ Of interest, plasma sCD30 levels were positively associated with NHL risk at 6-10 years ${ }^{9}$ and even $15-23$ years pre-diagnosis. ${ }^{11}$ Another small nested case-control study reported a significant 2.5 -fold increase in NHL risk in women with elevated soluble IL-2 receptor- $\alpha$ levels (sIL-2R $\alpha$; a marker of T-cell activation and IL-2 upregulation), and marginally significant increases in NHL risk in women with higher pre-diagnosis tumor necrosis factor (TNF)- $\alpha$ and soluble TNFreceptor-2 (sTNF-R2) levels. ${ }^{14}$ These findings collectively suggest that chronic B-cell stimulation has a role in lymphomagenesis in immunocompetent persons.

Our study aimed to further characterize pre-diagnosis plasma immune marker profiles associated with risk of HIV-unrelated NHL and its major histological subtypes in two large US cohorts. This study represents one of the largest populations with prospectively collected pre-diagnosis blood samples to investigate the association between numerous immune markers and NHL risk, including those with specific NHL subtypes that are often precluded due to small sample size, and to assess the independence of biomarker-NHL associations for multiple immune markers. ${ }^{1{ }^{1,12}}$ The long-term follow up of the study population also allowed for examination of the influence of time since blood draw on observed immune markerNHL associations, including an assessment of potential early markers of lymphomagenesis present ten years or more prior to diagnosis. The choice of immune markers was guided in part by the immune deregulation we sought to characterize and by reported findings in AIDS- or HIVunrelated NHL. We hypothesized that pre-diagnosis levels of immune markers indicative of B-cell activation or inflammation would be positively associated with risk of developing NHL and major NHL subtypes, and that the use of multi-marker models will enhance characterization of the immune milieu associated with NHL risk and suggest subtle differences by histological subtype.

\section{Methods}

\section{Study population}

The study population comprised Nurses' Health Study (NHS, all female) and Health Professionals Follow-up Study (HPFS, all male) participants with archived plasma (Online Supplementary Methods). ${ }^{15,16}$ Cancer diagnoses were identified via routine questionnaires or follow up after death ${ }^{17,18}$ and confirmed by medical record review or tumor registry linkage.

Participants provided written informed consent at blood collection. The study was conducted in accordance with the Declaration of Helsinki and approved by the Institutional Review Boards of the Brigham and Women's Hospital and Harvard T.H. Chan School of Public Health.

\section{Case and control selection}

We included all participants with confirmed incident NHL diagnosed three months or more after blood draw through $31^{\text {st }}$ December 2010 with no other cancer history. Study pathologists (JCA, SJR) classified NHL histological subtype ${ }^{19}$ according to World Health Organization ${ }^{20,21}$ and International Lymphoma Epidemiology (InterLymph) Consortium guidelines. ${ }^{22,23}$ We ana- lyzed common B-cell (B-)NHL subtypes individually [diffuse large B-cell lymphoma (DLBCL), follicular lymphoma (FL), and chronic lymphocytic leukemia/small lymphocytic lymphoma (CLL/SLL)], combined less common B-NHLs ("other B-NHL") and defined additional categories by cell type (T-NHL, B-NHL). We matched one control per case by sex (cohort), age, race, and blood draw details (Online Supplementary Methods).

\section{Biomarker assessment}

Assays were performed at the University of California, Los Angeles (LM, OMM), using multiplexed kits (Fluorokine ${ }^{\circledast} \mathrm{MAP}, \mathrm{R}$ \& D Systems, Minneapolis, MN, USA), a Bio-Plex 200 Luminex instrument and Bio-Plex analysis software (Bio-Rad, Hercules, CA, USA). Blinded laboratory personnel measured sCD30, sIL-2R $\alpha, B$ cell activating factor of the TNF family (BAFF, a B-cell stimulatory cytokine), CXCL13, sIL-6R $\alpha$, sGP130, sCD14, sTNF-R2, C-reactive protein (CRP), IL-6, IL-8, IL-10, and TNF- $\alpha$ concentration according to the manufacturer's directions (Online Supplementary Methods). We set TNF- $\alpha$, IL-8 and CXCL13 values to missing for samples with $>24$-hour processing delays (NHS: $n=35$; HPFS: $\mathrm{n}=23$ ). Analyte concentrations were natural log-transformed for all analyses. We observed similar measured biomarker concentrations for the NHS and HPFS (Online Supplementary Table S1) and pooled the data.

\section{Statistical analysis}

We conducted batch calibration to diminish the potential influence of laboratory batch-related variability on biomarker-NHL associations. ${ }^{24}$ Outlying biomarker values were identified using the Rosner extreme Studentized deviate method $^{25}$ and omitted from analyses of the marker.

The primary analysis assessed batch effect-corrected, log-transformed biomarker values continuously per Standard Deviation (SD) increase in concentration, with SD units calculated for logtransformed values in the pooled controls. We calculated Odds Ratios (OR) and 95\% Confidence Intervals (CI) for the association of each biomarker with NHL risk (overall and for DLBCL, FL, CLL/SLL, other B-NHL, all B-NHL and all T-NHL) using unconditional logistic regression. Models adjusted for all matching factors unless small cell counts precluded adjustment for race. We evaluated but did not observe confounding by body mass index (BMI) and autoimmune disease history.

We intended a priori to identify multi-marker profiles associated with NHL risk via mutual adjustment of models for biomarkers that were individually associated. We also examined models stratified by follow-up interval ( 0 to $<5,5$ to $<10, \geq 10$ years) and assessed heterogeneity by time period using the contrast test. ${ }^{26}$ The Online Supplementary Methods describe additional analyses designed post hoc.

\section{Results}

In total, 601 cases of NHL (345 NHS and 256 HPFS) were identified and individually matched to controls. Three cases were later excluded due to unconfirmed lymphoma status. The final analysis thus included 598 cases, including 114 DLBCL, $92 \mathrm{FL}, 165 \mathrm{CLL} / \mathrm{SLL}, 132$ other BNHL (4 Burkitt lymphoma, 19 lymphoplasmacytic lymphoma, 20 mantle cell lymphoma, 44 marginal zone lymphoma, 20 other B-NHL, and 25 unclassified B-NHL) and $30 \mathrm{~T}-\mathrm{NHL}$, and 601 controls. The study population was $96 \%$ Caucasian and $58 \%$ female. Cases and controls had similar covariable distributions, due in part to the matched design (Table 1). 
We omitted 109 individual biomarker measurements $(<1 \%$ of all measurements) with implausible outlying values (NHS: 72; HPFS: 37), the majority (90\%) of which were implausibly high for the particular marker. Omitted values ranged from one measure of IL-10 to 17 measures of IL-8. Spearman correlation coefficients ranged from -0.03 (IL-10 and CXCL13) to 0.58 (sIL-2R $\alpha$ and sCD30) (Online Supplementary Table S2).

\section{Individual immune marker models}

Multivariable analyses of individual log-transformed immune markers revealed significant associations for all NHL per SD increment of log-transformed sTNF-R2, sIL$2 \mathrm{R} \alpha, \mathrm{CXCL} 13, \mathrm{sCD} 30$ (all positive) and BAFF (inverse; Table 2). In subtype-specific analyses, sTNF-R2 levels were also positively associated with risk of all B-NHL, FL and CLL/SLL, while CXCL13 was positively associated with risk of all B-NHL, DLBCL and FL (Table 2). Levels of sIL-2R $\alpha$ and sCD30 were positively associated with every NHL subtype, including T-NHL. Of interest, the association of BAFF with a $17 \%$ decreased risk of all $\mathrm{NHL}$ appeared to be driven by CLL/SLL, for which risk decreased by $49 \%$ per SD increase in log-transformed BAFF levels (OR: 0.51; 95\%CI: 0.43, 0.62; $P<0.001$ ); BAFF was not associated with other NHL subtypes in singlemarker models. We did not observe significant or consistent associations for the remaining immune markers with risk of any NHL end point. Results from cohort-specific models did not suggest marked differences by sex for these associations (Online Supplementary Table S3).

\section{Multi-marker profiles}

In the model that mutually adjusted for the five logtransformed immune markers that had significant individ-

Table 1. Characteristics of non-Hodgkin lymphoma cases and matched controls from two prospective cohort studies.

\begin{tabular}{|c|c|c|c|}
\hline Variable & Cases & Controls & P* \\
\hline \multicolumn{4}{|l|}{ Cohort } \\
\hline NHS & $344(58 \%)$ & $345(57 \%)$ & 0.97 \\
\hline HPFS & $254(42 \%)$ & $256(43 \%)$ & \\
\hline Age, years, mean \pm SD & $60.8 \pm 8.1$ & $60.8 \pm 8.1$ & 0.87 \\
\hline \multicolumn{4}{|l|}{ Race/ethnicity } \\
\hline Caucasian & $573(96 \%)$ & $578(96 \%)$ & 0.75 \\
\hline Other & $25(4 \%)$ & $23(4 \%)$ & \\
\hline \multicolumn{4}{|l|}{ BMI at blood draw, $\mathrm{kg} / \mathrm{m}^{2}$} \\
\hline$<22.5$ & $138(23 \%)$ & $118(20 \%)$ & 0.58 \\
\hline $22.5-24.9$ & $139(23 \%)$ & $166(28 \%)$ & \\
\hline $25-29.9$ & $217(36 \%)$ & $219(36 \%)$ & \\
\hline$\geq 30$ & 74 (12\%) & 74 (12\%) & \\
\hline Missing & $30(5 \%)$ & $24(4 \%)$ & \\
\hline \multicolumn{4}{|c|}{ BMI in young adulthood, $\mathrm{kg} / \mathrm{m}^{2}$} \\
\hline$<18.5$ & $44(7 \%)$ & $54(9 \%)$ & 0.31 \\
\hline $18.5-22.4$ & $298(50 \%)$ & $299(50 \%)$ & \\
\hline $22.5-24.9$ & $126(21 \%)$ & $112(19 \%)$ & \\
\hline$\geq 25$ & $106(18 \%)$ & $101(17 \%)$ & \\
\hline Missing & $24(4 \%)$ & $35(6 \%)$ & \\
\hline \multicolumn{4}{|l|}{ Autoimmune disease $^{\dagger}$} \\
\hline Yes & $97(16 \%)$ & $104(17 \%)$ & 0.62 \\
\hline No & $501(84 \%)$ & $497(83 \%)$ & \\
\hline \multicolumn{4}{|c|}{ Years from blood draw to index date, } \\
\hline Mean \pm SD & $9.6 \pm 5.6$ & $9.6 \pm 5.6$ & 0.99 \\
\hline \multicolumn{4}{|c|}{ Cell type/histological subtype of $\mathrm{NHL}^{\ddagger}$} \\
\hline B-NHL & $503(84 \%)$ & & \\
\hline DLBCL & $114(19 \%)$ & & \\
\hline Follicular lymphoma & $92(15 \%)$ & & \\
\hline CLL/SLL & $165(28 \%)$ & & \\
\hline Other B-cell subtypes ${ }^{\S}$ & $132(22 \%)$ & & \\
\hline T-NHL & $30(5 \%)$ & & \\
\hline
\end{tabular}


Table 2. Associations between pre-diagnosis concentrations of 13 individual immune markers and risk of non-Hodgkin lymphoma (NHL), overall and by major histological subtype.

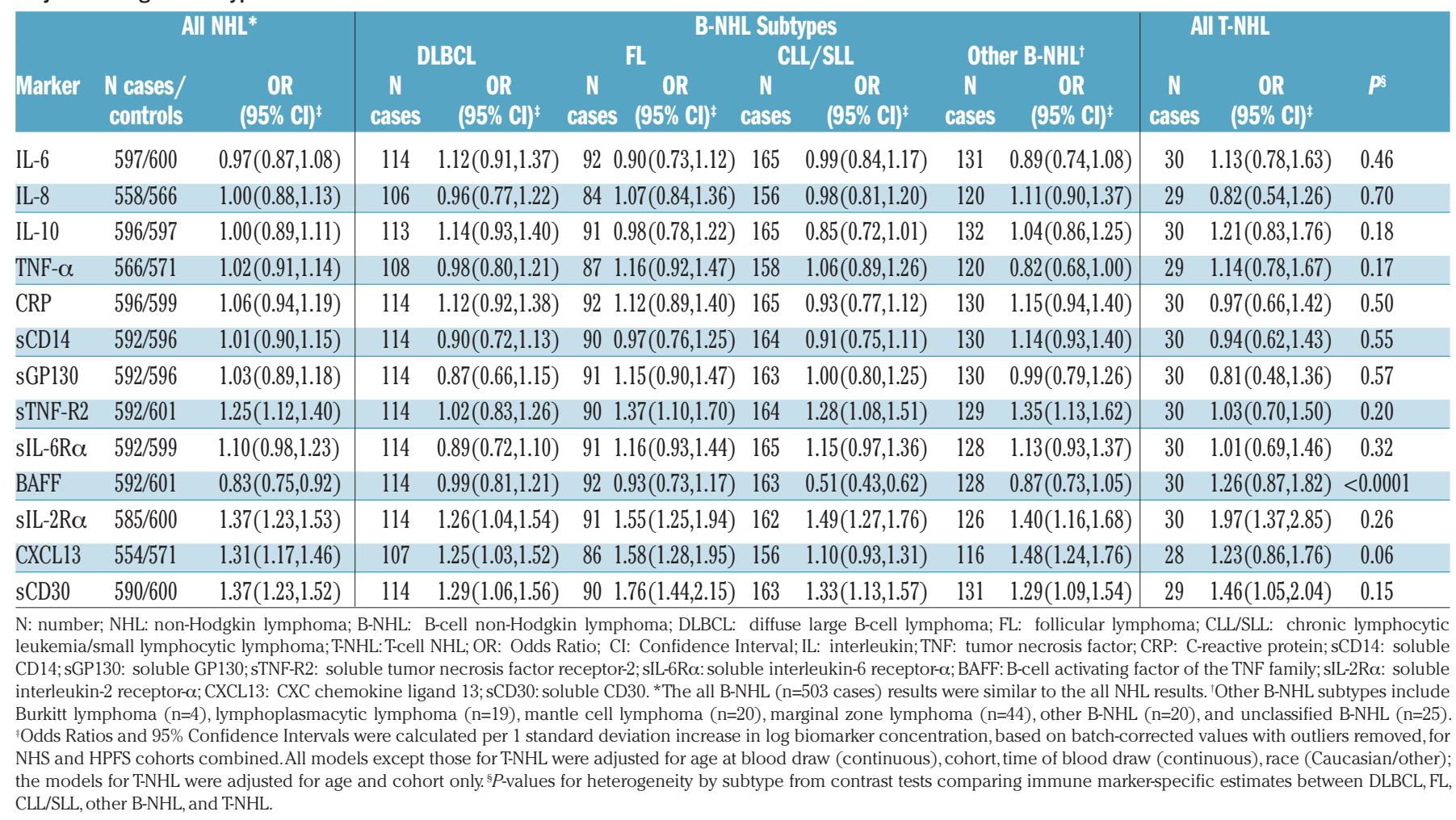

ual associations with NHL end points (sTNF-R2, sIL-2R $\alpha$, CXCL13, sCD30, BAFF), sIL-2R $\alpha$, CXCL13 and sCD30 remained significantly associated with a $17-24 \%$ increased risk, and BAFF with a $26 \%$ decreased risk of all NHL per SD increase in log concentration, while sTNF-R2 was no longer significantly associated (Table 3). Results for all B-NHL risk were similar to those for all NHL, whereas mutual adjustment attenuated all the immune marker associations with DLBCL. In the multi-marker model of FL risk, sCD30 and BAFF remained independently associated, with a borderline association noted for CXCL13 (Table 4). In the multi-marker model of CLL/SLL risk, sIL-2R $\alpha$ was significantly associated with a $50 \%$ increase (95\% CI: 1.18-1.90), and BAFF with a significant $53 \%$ reduction $(95 \% \mathrm{CI}: 0.38,0.58)$, per SD increase in log concentration. Lastly, only sIL-2R $\alpha$ was independently associated with T-NHL risk (OR per SD increase in log concentration: 1.96 ; $95 \% \mathrm{CI}: 1.22,3.13)$ in mutually adjusted models.

The 5-marker models using the polytomous logistic regression (PLR) approach yielded essentially the same effect estimates as described above for biomarker associations with the NHL end points for the full follow-up period (Online Supplementary Tables S4 and S5). sTNF-R2 had significantly different associations with B-NHL and TNHL ( $P$-value for heterogeneity by subtype $=0.04)$ (Online Supplementary Table S4); the associations of CXCL13 and BAFF with individual B-NHL subtypes also showed evidence of significant heterogeneity ( $P$-values for heterogeneity by subtype $=0.007$ and $<0.0001$, respectively) (Online Supplementary Table S5).

In the covariable-adjusted multi-marker models containing restricted cubic splines, there was evidence of non-lin- earity for two biomarkers, CXCL13 and BAFF, in their associations with risk of aggregated end points (all NHL, B-NHL and other B-NHL; P-value tests for significance of the curve $<0.05$ ), but not for biomarker associations with individual B-NHL subtypes or T-NHL.

In alternative models using semi-automatic stepwise selection, the final models for all NHL and all B-NHL included sIL-2R $\alpha$, CXCL13 and sCD30, which were positively associated with risk, as well as BAFF, which was inversely associated (Online Supplementary Table S6). In comparison, for DLBCL and FL, the stepwise procedure selected only sCD30 ( $P=0.004$ and $<0.0001$, respectively), and for T-NHL the procedure selected only sIL-2R $\alpha$ $(P=0.002)$ as independently (positively) associated with risk. Of interest, the stepwise procedure identified four immune markers independently associated with risk of CLL/SLL, including BAFF and IL-10 with significant inverse associations and sIL-2R $\alpha$ with a significant positive association. The stepwise procedure identified three immune markers associated with the combined category of other B-NHL subtypes, including significant positive associations for CXCL13 and sIL-2R $\alpha$, and a significant inverse association for BAFF.

In the model that included all 13 immune markers, sIL$2 \mathrm{R} \alpha$, CXCL13, and sCD30 again had strong positive associations with risk of all NHL and all B-NHL (Table 5). In the CLL/SLL-specific model, we observed a significant inverse association with risk for BAFF and also for sCD14 and IL-10, and a positive association with sIL2-R $\alpha$. BAFF was also significantly inversely associated with FL risk, while sCD30 was significantly positively associated with FL risk. Only sIL-2R $\alpha$ was significantly associated with an increased risk of T-NHL. We observed suggestive positive 
Table 3. Independent associations of multiple pre-diagnosis plasma immune markers with risk of non-Hodgkin lymphoma (NHL), overall and by B- or T-cell type of origin, for the complete follow-up period and stratified by years of follow up.

\begin{tabular}{|c|c|c|c|c|c|c|c|c|c|}
\hline \multirow[b]{3}{*}{ Marker } & \multirow{2}{*}{\multicolumn{2}{|c|}{ Complete follow-up period }} & \multicolumn{6}{|c|}{ Years from blood draw to diagnosis/index date } & \multirow[b]{3}{*}{$P \neq$} \\
\hline & & & 0 to & s than 5 & 5 to less & than 10 & 10 & r more & \\
\hline & $\begin{array}{l}\text { N cases/ } \\
\text { controls }\end{array}$ & $\begin{array}{c}\text { OR } \\
\text { (95\% CI) } \\
\text { per 1-SD *,t }\end{array}$ & $\begin{array}{l}\text { N cases/ } \\
\text { controls }\end{array}$ & $\begin{array}{c}\text { OR } \\
\text { (95\% CI) } \\
\text { per 1-SD *,t }\end{array}$ & $\begin{array}{l}\text { N cases/ } \\
\text { controls }\end{array}$ & $\begin{array}{c}\text { OR } \\
\text { (95\% CI) } \\
\text { per 1-SD *,t }\end{array}$ & $\begin{array}{l}\text { N cases/ } \\
\text { controls }\end{array}$ & $\begin{array}{c}\text { OR } \\
\text { (95\% CI) } \\
\text { per 1-SD *,t }\end{array}$ & \\
\hline
\end{tabular}

All NHL

\begin{tabular}{|c|c|c|c|c|c|c|c|c|}
\hline sTNF-R2 & $542 / 571$ & $1.05(0.91,1.21)$ & $133 / 140$ & $0.83(0.60,1.14)$ & 149/162 $1.02(0.77,1.35)$ & $260 / 267$ & $1.18(0.95,1.46)$ & 0.20 \\
\hline sIL-2R $\alpha$ & $542 / 571$ & $1.20(1.03,1.39)$ & $133 / 140$ & $1.52(1.09,2.11)$ & $149 / 1621.16(0.88,1.53)$ & $260 / 267$ & $1.11(0.88,1.39)$ & 0.28 \\
\hline CXCL13 & $542 / 571$ & $1.17(1.03,1.32)$ & $133 / 140$ & $1.00(0.78,1.29)$ & $149 / 1621.30(1.03,1.62)$ & $260 / 267$ & $1.21(1.01,1.46)$ & 0.32 \\
\hline sCD30 & $542 / 571$ & $1.24(1.06,1.45)$ & $133 / 140$ & $1.52(1.09,2.13)$ & $149 / 1621.43(1.07,1.90)$ & $260 / 267$ & $0.98(0.78,1.23)$ & 0.02 \\
\hline BAFF & $542 / 571$ & $0.74(0.66,0.83)$ & $133 / 140$ & $0.73(0.59,0.91)$ & $149 / 1620.61(0.48,0.78)$ & $260 / 267$ & $0.83(0.69,1.00)$ & 0.15 \\
\hline \multicolumn{9}{|l|}{ All B-NHL } \\
\hline sTNF-R2 & $454 / 570$ & $1.07(0.92,1.25)$ & $110 / 140$ & $0.88(0.63,1.23)$ & $118 / 1611.15(0.86,1.54)$ & $226 / 267$ & $1.13(0.90,1.42)$ & 0.40 \\
\hline sIL-2R $\alpha$ & $454 / 570$ & $1.20(1.03,1.41)$ & $110 / 140$ & $1.51(1.06,2.14)$ & $118 / 1611.12(0.84,1.49)$ & $226 / 267$ & $1.15(0.91,1.46)$ & 0.38 \\
\hline CXCL13 & $454 / 570$ & $1.13(1.00,1.29)$ & $110 / 140$ & $0.96(0.74,1.25)$ & $118 / 1611.17(0.92,1.49)$ & $226 / 267$ & $1.24(1.02,1.50)$ & 0.31 \\
\hline sCD30 & $454 / 570$ & $1.24(1.05,1.46)$ & $110 / 140$ & $1.59(1.10,2.28)$ & $118 / 1611.58(1.14,2.20)$ & $226 / 267$ & $0.96(0.75,1.22)$ & 0.02 \\
\hline BAFF & $454 / 570$ & $0.73(0.64,0.83)$ & $110 / 140$ & $0.67(0.53,0.84)$ & $118 / 1610.64(0.50,0.81)$ & $226 / 267$ & $0.84(0.69,1.02)$ & 0.14 \\
\hline \multicolumn{9}{|l|}{ All T-NHL } \\
\hline sTNF-R2 & $28 / 569$ & $0.62(0.37,1.03)$ & $11 / 140$ & $0.44(0.17,1.19)$ & $10 / 160 \quad 0.65(0.27,1.58)$ & $7 / 267$ & $0.73(0.24,2.21)$ & 0.78 \\
\hline sIL-2R $\alpha$ & $28 / 569$ & $1.96(1.22,3.13)$ & $11 / 140$ & $2.10(0.95,4.68)$ & $10 / 1602.20(0.93,5.20)$ & $7 / 267$ & $1.04(0.36,3.00)$ & 0.50 \\
\hline CXCL13 & $28 / 569$ & $1.11(0.75,1.65)$ & $11 / 140$ & $1.03(0.48,2.22)$ & $10 / 1601.37(0.78,2.42)$ & $7 / 267$ & $0.57(0.24,1.37)$ & 0.26 \\
\hline sCD30 & $28 / 569$ & $1.33(0.84,2.10)$ & $11 / 140$ & $1.68(0.69,4.08)$ & $10 / 1601.34(0.56,3.21)$ & $7 / 267$ & $1.77(0.70,4.43)$ & 0.90 \\
\hline BAFF & $28 / 569$ & $0.88(0.58,1.32)$ & $11 / 140$ & $0.93(0.52,1.67)$ & $10 / 160 \quad 0.55(0.24,1.28)$ & $7 / 267$ & $1.68(0.64,4.44)$ & 0.23 \\
\hline
\end{tabular}

N: number; NHL: non-Hodgkin lymphoma; B-NHL: B-cell NHL;T-NHL: T-cell NHL; OR: Odds Ratio; CI: Confidence Interval; SD: Standard Deviation; sTNF-R2: soluble tumor necrosis factor receptor-2; sIL-2R $\alpha$ : soluble interleukin-2 receptor- $\alpha$; CXCL13: CXC chemokine ligand 13; sCD30: soluble CD30; BAFF: B-cell activating factor of the TNF family. *Models were adjusted for age at blood draw (continuous), cohort (sex), time of blood draw (continuous) and race/ethnicity (Caucasian, non-Caucasian) and were mutually adjusted for all markers listed, except that models for all T-NHL were not adjusted for race. ${ }^{\top}$ Odds Ratios and $95 \%$ Confidence Intervals were calculated per 1-standard deviation increase in batch effect-corrected, log-transformed values (with cohort-specific outliers excluded) from the Nurses' Health Study and Health Professionals Follow-up Study combined. $P_{\text {-values }}$ from tests for heterogeneity comparing immune marker-specific estimates across time strata.

associations of DLBCL risk with IL-6, CXCL13 and sCD30 in this 13-marker model.

\section{Time-stratified analyses}

The analyses stratified by time between blood draw and diagnosis/index date suggested that the individual biomarker associations with all NHL (Online Supplementary Table S7) and with NHL subtypes (Online Supplementary Table S8) varied somewhat by length of time after blood draw but did not strongly implicate any additional immune marker-NHL associations. The time-stratified 5marker models (Table 3 ) also suggested variability by follow-up interval in the independent associations of those immune markers with future NHL risk. For example, the association of sIL-2R $\alpha$ with risk of all NHL appeared to be restricted to a shorter-term interval, specifically within five years of blood draw (OR: $1.52,95 \%$ CI: 1.09, 2.11) (Table 3), whereas significant associations of CXCL13 with risk of all NHL were evident only five or more years after blood collection (5-<10 years; OR: $1.23,95 \%$ CI: 1.00 , 1.52; and $\geq 10$ years; OR: $1.21,95 \%$ CI: $1.01,1.45)$. sCD30 was most strongly associated with all NHL risk within ten years of blood draw, while BAFF was consistently inversely associated with all NHL across time periods. Of note, in subtype-specific time-stratified analyses, sCD30 levels were strongly positively associated with risk of FL within five years of blood draw (OR: 4.85, 95\% CI: 2.02, 11.61), and the association decreased in magnitude with increasing follow-up time. In CLL/SLL-specific models, elevated sIL-2R $\alpha$ was associated with a nearly 4-fold increased risk within five years of blood draw (OR: 3.71, 95\%CI: 1.77, 7.76) but had no clear association with longer-term CLL/SLL risk. In contrast, BAFF had significant inverse associations with risk of CLL/SLL in all pre-diagnosis time periods, albeit with particularly strong associations with risk of CLL/SLL within five or ten years of blood draw (Table 4). When modeled using PLR, the effect estimates were virtually the same for time period-specific biomarker associations, both for the aggregated and the individual NHL end points (Online Supplementary Tables S4 and S5). The most prominent differences between the two approaches for assessing heterogeneity by time period (PLR with interaction terms vs. time-stratified unconditional logistic regression) pertained to the statistical significance of apparent heterogeneity by follow-up period for the associations of sTNF-R2 with all B-NHL and FL. For example, for the association of sTNF-R2 with all B-NHL, the $P$-value for heterogeneity by follow-up time was 0.04 for the cross-product term in PLR (Online Supplementary Table S4) and 0.40 for the main model contrast test (Table $3)$. For the association of sTNF-R2 with FL, the $P$-value for heterogeneity by time period was 0.0007 for the crossproduct term in PLR (Online Supplementary Table S5) and 0.11 for the main model contrast test (Table 4). Time-strat- 
Table 4. Independent associations of multiple pre-diagnosis plasma immune markers with risk of non-Hodgkin lymphoma (NHL) by major histological subtype of B-cell NHL, for the complete follow-up period and stratified by years of follow up.

\begin{tabular}{|c|c|c|c|c|c|c|c|c|c|}
\hline \multirow[b]{3}{*}{ Marker } & \multicolumn{9}{|c|}{ Years from blood draw to diagnosis/index date } \\
\hline & Complet & ow-up period & 0 to & than 5 & 5 to les & han 10 & & ore & \\
\hline & $\begin{array}{l}\text { N cases/ } \\
\text { controls }\end{array}$ & $\begin{array}{c}\text { OR } \\
\text { (95\% CI) } \\
\text { per 1-SD*,t }\end{array}$ & $\begin{array}{l}\text { N cases/ } \\
\text { controls }\end{array}$ & $\begin{array}{c}\text { OR } \\
\text { (95\% CI) } \\
\text { per 1-SD*, }\end{array}$ & $\begin{array}{l}N \text { cases/ } \\
\text { controls }\end{array}$ & $\begin{array}{c}\text { OR } \\
\text { (95\% CI) } \\
\text { per 1-SD*,t }\end{array}$ & $\begin{array}{l}\text { N cases/ } \\
\text { controls }\end{array}$ & $\begin{array}{c}\text { OR } \\
\text { (95\% CI) } \\
\text { per 1-SD*, }\end{array}$ & $p^{*}$ \\
\hline
\end{tabular}

DLBCL

\begin{tabular}{|c|c|c|c|c|c|c|c|c|c|}
\hline sTNF-R2 & $107 / 570$ & $0.81(0.62,1.07)$ & $25 / 140$ & $0.61(0.34,1.10)$ & $25 / 161$ & $1.05(0.60,1.85)$ & $57 / 267$ & $0.83(0.56,1.24)$ & 0.42 \\
\hline sIL-2R $\alpha$ & $107 / 570$ & $1.18(0.91,1.53)$ & $25 / 140$ & $1.83(1.00,3.37)$ & $25 / 161$ & $1.19(0.67,2.11)$ & $57 / 267$ & $1.09(0.75,1.58)$ & 0.35 \\
\hline CXCL13 & $107 / 570$ & $1.17(0.95,1.45)$ & $25 / 140$ & $0.71(0.43,1.19)$ & $25 / 161$ & $1.42(0.95,2.12)$ & $57 / 267$ & $1.30(0.95,1.79)$ & 0.09 \\
\hline sCD30 & $107 / 570$ & $1.23(0.95,1.59)$ & $25 / 140$ & $0.90(0.48,1.67)$ & $25 / 161$ & $1.76(1.07,2.89)$ & $57 / 267$ & $1.09(0.75,1.59)$ & 0.19 \\
\hline BAFF & $107 / 570$ & $0.95(0.76,1.18)$ & $25 / 140$ & $0.96(0.59,1.55)$ & $25 / 161$ & $0.69(0.44,1.08)$ & $57 / 267$ & $1.08(0.78,1.50)$ & 0.27 \\
\hline \multicolumn{10}{|l|}{$\mathrm{FL}$} \\
\hline sTNF-R2 & $83 / 569$ & $1.03(0.77,1.38)$ & $18 / 140$ & $0.45(0.16,1.25)$ & $22 / 160$ & $0.95(0.53,1.69)$ & $43 / 267$ & $1.35(0.93,1.96)$ & 0.11 \\
\hline sIL-2R $\alpha$ & $83 / 569$ & $1.06(0.78,1.46)$ & $18 / 140$ & $0.93(0.35,2.44)$ & $22 / 160$ & $1.09(0.63,1.89)$ & $43 / 267$ & $1.09(0.70,1.68)$ & 0.95 \\
\hline CXCL13 & $83 / 569$ & $1.24(0.98,1.58)$ & $18 / 140$ & $1.10(0.58,2.06)$ & $22 / 160$ & $1.12(0.72,1.75)$ & $43 / 267$ & $1.48(1.03,2.13)$ & 0.56 \\
\hline sCD30 & $83 / 569$ & $1.69(1.26,2.26)$ & $18 / 140$ & $4.85(2.02,11.61)$ & $22 / 160$ & $1.88(1.04,3.40)$ & $43 / 267$ & $1.06(0.68,1.64)$ & 0.007 \\
\hline BAFF & $83 / 569$ & $0.76(0.59,0.98)$ & $18 / 140$ & $0.70(0.38,1.30)$ & $22 / 160$ & $0.72(0.41,1.24)$ & $43 / 267$ & $0.78(0.55,1.12)$ & 0.94 \\
\hline \multicolumn{10}{|l|}{ CLL/SLL } \\
\hline sTNF-R2 & $153 / 569$ & $1.21(0.96,1.52)$ & $36 / 140$ & $0.98(0.49,1.93)$ & $44 / 160$ & $1.27(0.81,1.98)$ & $73 / 267$ & $1.16(0.85,1.58)$ & 0.82 \\
\hline sIL-2R $\alpha$ & $153 / 569$ & $1.50(1.18,1.90)$ & $36 / 140$ & $3.71(1.77,7.76)$ & $44 / 160$ & $1.39(0.90,2.15)$ & $73 / 267$ & $1.26(0.87,1.83)$ & 0.04 \\
\hline CXCL13 & $153 / 569$ & $0.90(0.74,1.10)$ & $36 / 140$ & $0.78(0.48,1.27)$ & $44 / 160$ & $0.80(0.54,1.20)$ & $73 / 267$ & $1.04(0.77,1.41)$ & 0.48 \\
\hline sCD30 & $153 / 569$ & $1.15(0.89,1.48)$ & $36 / 140$ & $1.43(0.71,2.87)$ & $44 / 160$ & $1.54(0.96,2.46)$ & $73 / 267$ & $0.90(0.62,1.30)$ & 0.17 \\
\hline BAFF & $153 / 569$ & $0.47(0.38,0.58)$ & $36 / 140$ & $0.32(0.19,0.53)$ & $44 / 160$ & $0.39(0.25,0.61)$ & $73 / 267$ & $0.63(0.46,0.86)$ & 0.05 \\
\hline \multicolumn{10}{|c|}{ Other B-NHL ${ }^{8}$} \\
\hline sTNF-R2 & $111 / 569$ & $1.17(0.92,1.49)$ & $31 / 140$ & $1.28(0.78,2.12)$ & $27 / 160$ & $1.17(0.73,1.90)$ & $53 / 267$ & $1.11(0.77,1.61)$ & 0.90 \\
\hline sIL-2R $\alpha$ & $111 / 569$ & $1.15(0.89,1.48)$ & $31 / 140$ & $1.07(0.62,1.83)$ & $27 / 160$ & $1.05(0.63,1.74)$ & $53 / 267$ & $1.29(0.87,1.91)$ & 0.77 \\
\hline CXCL13 & $111 / 569$ & $1.45(1.19,1.77)$ & $31 / 140$ & $1.50(0.98,2.30)$ & $27 / 160$ & $1.52(1.07,2.17)$ & $53 / 267$ & $1.36(0.98,1.88)$ & 0.88 \\
\hline sCD30 & $111 / 569$ & $1.08(0.82,1.41)$ & $31 / 140$ & $1.28(0.73,2.26)$ & $27 / 160$ & $1.37(0.81,2.32)$ & $53 / 267$ & $0.79(0.52,1.21)$ & 0.20 \\
\hline BAFF & $111 / 569$ & $0.78(0.64,0.95)$ & $31 / 140$ & $0.74(0.53,1.04)$ & $27 / 160$ & $0.72(0.48,1.07)$ & $53 / 267$ & $0.89(0.64,1.24)$ & 0.66 \\
\hline
\end{tabular}

N: number; DLBCL: diffuse large B-cell lymphoma; FL: follicular lymphoma; CLL/SLL: chronic lymphocytic leukemia/small lymphocytic lymphoma; B-NHL: B-cell NHL; OR: Odds Ratio; CI: Confidence Interval; SD: Standard Deviation; sTNF-R2: soluble tumor necrosis factor receptor-2; sIL-2R $\alpha$ : soluble interleukin-2 receptor- $\alpha$; CXCL13: CXC chemokine ligand 13; sCD30: soluble CD30; BAFF: B-cell activating factor of the TNF family. *All models were adjusted for age at blood draw (continuous), cohort (sex), time of blood draw (continuous) and race/ethnicity (Caucasian, non-Caucasian), and were mutually adjusted for all markers listed, except that models for other B-NHL were not adjusted for race. Odds Ratios and 95\% Confidence Intervals were calculated per 1-standard deviation increase in batch effect-corrected, log-transformed values (with cohort-specific outliers excluded) from the Nurses' Health Study and Health Professionals Follow-up Study combined. ${ }^{*} P$-values from tests for heterogeneity comparing immune marker-specific estimates across time strata. ${ }^{\circledR}$ Other B-NHL subtypes included Burkitt lymphoma $(\mathrm{n}=4)$, lymphoplasmacytic lymphoma $(\mathrm{n}=19)$, mantle cell lymphoma ( $\left.=20\right)$, marginal zone lymphoma ( $\mathrm{n}=39)$, other B-NHL $(\mathrm{n}=20)$, and unclassified B-NHL $(\mathrm{n}=25)$.

ified results for the multi-marker models identified with stepwise selection largely agreed with the main results described above (Online Supplementary Table S6).

\section{Discussion}

In this pooled analysis within the NHS and HPFS cohorts, we observed significant associations between NHL risk and pre-diagnosis levels of specific plasma immune markers, including a novel, inverse association between levels of BAFF and risk of CLL/SLL. Positive associations between levels of sIL-2R $\alpha$, CXCL13, and SCD30 and risk of all NHL and all B-NHL, as well as the inverse association of BAFF with risk of all NHL and CLL/SLL, were consistent and independent across several analytical approaches to constructing a multi-marker profile associated with risk. In contrast, the individual positive associations noted for sTNF-R2 with risk of all NHL and some B-
NHL end points were attenuated upon adjustment for other immune markers, suggesting a lack of independence in the association between sTNF-R2 levels and NHL risk. Manual selection and automated stepwise selection of multi-marker profiles yielded fairly consistent results for all NHL, but also some differences for individual histological subtypes, particularly for CLL/SLL. We also observed some variation in the associations between NHL risk and immune markers by time between blood draw and diagnosis.

Our findings are in agreement with previous studies reporting associations between elevated CXCL13 and/or sCD30 levels and increased NHL risk in HIV-positive and immunocompetent populations, including several reports analyzing blood samples taken many years prior to NHL diagnosis. $2,3,8-13$ In the Alpha-Tocopherol, Beta-Carotene Cancer Prevention Study, Purdue et al. ${ }^{11}$ prospectively investigated multi-marker models similar to those in our analysis and observed independent positive associations 
Table 5. Associations of pre-diagnosis plasma immune markers with risk of non-Hodgkin lymphoma (NHL), with mutual adjustment for all thirteen immune markers, for all NHL and by major histological subtype.

\begin{tabular}{|c|c|c|c|c|c|c|c|c|c|c|c|c|}
\hline & & NHL & & & & B-NHL Subt & ypes & & & & & All T-NHL \\
\hline & & & & DLBCL & & FL & & LL/SLL & & ler B-NHL” & & \\
\hline Marker & $\begin{array}{l}\text { N Cases/ } \\
\text { Controls }\end{array}$ & $\begin{array}{c}\text { OR } \\
(95 \% \text { CI) } \\
\text { per 1-SD *,t }\end{array}$ & N cases & $\begin{array}{c}\text { OR } \\
(95 \% \text { CI) } \\
\text { per } 1-S D^{*, \dagger}\end{array}$ & $\begin{array}{c}\text { N } \\
\text { cases }\end{array}$ & $\begin{array}{c}\text { OR } \\
(95 \% \text { CI) } \\
\text { per 1-SD*, }\end{array}$ & $\begin{array}{c}\mathrm{N} \\
\text { cases }\end{array}$ & $\begin{array}{c}\text { OR } \\
(95 \% \text { CI) } \\
\text { per 1-SD }{ }^{*, \dagger}\end{array}$ & $\begin{array}{c}\text { N } \\
\text { cases }\end{array}$ & $\begin{array}{c}\text { OR } \\
(95 \% \text { CI) } \\
\text { per 1-SD*, }\end{array}$ & $\begin{array}{c}\text { N } \\
\text { cases }\end{array}$ & $\begin{array}{c}\text { OR } \\
\text { (95\% CI) } \\
\text { per 1-SD*,† }\end{array}$ \\
\hline IL-6 & $523 / 550$ & $1.06(0.93,1.21)$ & 104 & $1.22(0.96,1.55)$ & 78 & $0.86(0.66,1.12)$ & 150 & $1.10(0.89,1.37)$ & 106 & $1.03(0.81,1.31)$ & 28 & $1.10(0.71,1.73)$ \\
\hline IL-8 & $523 / 550$ & $0.96(0.83,1.10)$ & 104 & $0.92(0.72,1.18)$ & 78 & $0.94(0.71,1.24)$ & 150 & $1.00(0.79,1.26)$ & 106 & $1.11(0.88,1.38)$ & 28 & $0.65(0.40,1.07)$ \\
\hline IL-10 & $523 / 550$ & $0.95(0.84,1.07)$ & 104 & $1.14(0.91,1.43)$ & 78 & $0.93(0.72,1.22)$ & 150 & $0.80(0.65,0.99)$ & 106 & $0.99(0.79,1.23)$ & 28 & $1.19(0.77,1.82)$ \\
\hline TNF- $\alpha$ & $523 / 550$ & $1.00(0.87,1.15)$ & 104 & $0.84(0.66,1.08)$ & 78 & $1.09(0.81,1.46)$ & 150 & $1.09(0.87,1.37)$ & 106 & $0.86(0.67,1.10)$ & 28 & $1.18(0.74,1.89)$ \\
\hline CRP & $523 / 550$ & $1.00(0.87,1.15)$ & 104 & $1.07(0.84,1.37)$ & 78 & $1.29(0.96,1.72)$ & 150 & $0.82(0.65,1.04)$ & 106 & $1.00(0.77,1.29)$ & 28 & $0.85(0.54,1.34)$ \\
\hline sCD14 & $523 / 550$ & $0.84(0.71,1.00)$ & 104 & $0.82(0.61,1.11)$ & 78 & $0.84(0.61,1.18)$ & 150 & $0.68(0.51,0.89)$ & 106 & $0.92(0.68,1.24)$ & 28 & $0.90(0.51,1.56)$ \\
\hline sGP130 & $523 / 550$ & $1.06(0.85,1.32)$ & 104 & $1.05(0.71,1.55)$ & 78 & $1.20(0.78,1.86)$ & 150 & $1.11(0.78,1.58)$ & 106 & $0.84(0.57,1.22)$ & 28 & $0.76(0.36,1.60)$ \\
\hline sTNF-R2 & $523 / 550$ & $1.06(0.86,1.31)$ & 104 & $0.96(0.67,1.37)$ & 78 & $0.88(0.57,1.36)$ & 150 & $1.33(0.94,1.87)$ & 106 & $1.16(0.81,1.65)$ & 28 & $0.75(0.38,1.46)$ \\
\hline sIL-6R $\alpha$ & $523 / 550$ & $1.03(0.89,1.20)$ & 104 & $0.86(0.66,1.12)$ & 78 & $1.02(0.74,1.40)$ & 150 & $1.14(0.90,1.45)$ & 106 & $1.19(0.91,1.56)$ & 28 & $1.11(0.67,1.84)$ \\
\hline BAFF & $523 / 550$ & $0.73(0.64,0.82)$ & 104 & $0.98(0.78,1.23)$ & 78 & $0.74(0.56,0.97)$ & 150 & $0.46(0.37,0.57)$ & 106 & $0.76(0.62,0.94)$ & 28 & $0.88(0.58,1.34)$ \\
\hline sIL-2R $\alpha$ & $523 / 550$ & $1.19(1.02,1.40)$ & 104 & $1.09(0.82,1.44)$ & 78 & $1.09(0.77,1.54)$ & 150 & $1.59(1.23,2.06)$ & 106 & $1.10(0.83,1.46)$ & 28 & $1.95(1.22,3.10)$ \\
\hline CXCL13 & $523 / 550$ & $1.18(1.04,1.34)$ & 104 & $1.20(0.96,1.49)$ & 78 & $1.22(0.94,1.59)$ & 150 & $0.92(0.74,1.13)$ & 106 & $1.45(1.18,1.78)$ & 28 & $1.20(0.80,1.82)$ \\
\hline sCD30 & $523 / 550$ & $1.26(1.06,1.48)$ & 104 & $1.27(0.96,1.66)$ & 78 & $1.80(1.30,2.50)$ & 150 & $1.10(0.84,1.45)$ & 106 & $1.13(0.85,1.52)$ & 28 & $1.25(0.76,2.05)$ \\
\hline$H$ & $N H \cdot B_{-}$ & tand & & Odds Ratio $\mathrm{Cl}$ & & & & ge & & - & 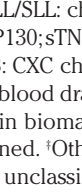 & onic lymph \\
\hline
\end{tabular}

for sCD30 with risk of NHL and DLBCL when adjusted for other biomarkers. Those observations were detectable more than 15 years prior to diagnosis. Also similar to our findings, positive associations observed for sTNF-R2 with NHL did not persist upon adjustment for other immune markers. ${ }^{11}$ In a prospective analysis in the Prostate, Lung, Colorectal and Ovarian Cancer Screening Trial, individual associations of CXCL13 and sTNF-R2 with NHL both remained significant with mutual adjustment, with correction for multiple comparisons and with restriction to samples collected 8-13 years prior to diagnosis. ${ }^{10}$

We observed an unexpected yet consistently strong inverse association between BAFF levels and CLL/SLL risk. BAFF is a member of the TNF family involved with B-cell survival and maturation. ${ }^{27}$ Pre-diagnosis serum BAFF concentrations were positively associated with AIDS-NHL, and BAFF overproduction has been associated with systemic autoimmune diseases, including systemic lupus erythematosus and Sjögren syndrome, ${ }^{28,29}$ which are associated with an increased risk of NHL in HIV-negative persons. ${ }^{30,31}$ However, systemic autoimmune disorders in HIV-negative individuals appear to be preferentially associated with NHL subtypes with a different natural history than CLL/SLL. ${ }^{30,32}$ Nonetheless, CLL cells are known to express multiple BAFF receptors (including TNFRSF13B, TNFRS13C and TNFRSF17), ${ }^{33}$ and the inverse association that we observed is biologically plausible if considered indicative of rapid uptake of circulating BAFF by nascent CLL/SLL clones, ${ }^{34}$ reflecting subclinical progression of an indolent tumor whose natural course may extend multiple decades. Consistent with this interpretation, several clinical studies have observed lower levels of BAFF in sera from CLL/SLL patients than in healthy controls..$^{35-37}$ The mechanism for the latter findings is unknown; our observation suggests that those underlying physiological processes may commence early in CLL pathogenesis, even ten or more years pre-diagnosis. Concurrent measurement of soluble BAFF receptors and study of cell surface expression of those molecules and classification of cases into prognostic subgroups were not feasible for the present study. Confirmation of the present findings is warranted in larger populations with specimens suitable for determining cell surface marker or gene/protein expression. Additionally, prospective studies in patients with monoclonal B-lymphocytosis would be informative to evaluate whether circulating BAFF levels can enhance risk stratification for progression to malignancy. ${ }^{38}$

We also observed significant associations of elevated sIL-2R $\alpha$ levels with increased risks of all NHL, B-NHL, DLBCL, CLL/SLL and T-NHL, primarily within five years of blood draw. One other study reported a positive association between sIL-2R $\alpha$ levels and NHL risk in an HIVnegative population with prospective blood collection that persisted after incorporating lag-times greater than two years. ${ }^{14}$ Of note, comparatively high sIL-2R $\alpha$ levels at diagnosis were also associated with poor prognosis in patients with NHL. ${ }^{39-41}$ Biologically, sIL-2R $\alpha$ and sCD30 are highly correlated ( $r=0.58$ in this study), and both can indicate Band T-cell activation; ${ }^{42}$ in the present analysis, both markers remained independently associated with a significant increased risk of all NHL and all B-NHL after mutual adjustment. In contrast, only sIL-2R $\alpha$ was significantly associated with an increased risk of T-NHL in the multimarker models, although small sample size ( $n=30$ cases) limited statistical power to detect significant independent associations for more strongly correlated biomarkers. Of 
interest, we observed the strongest positive associations of sIL-2R $\alpha$ with T-NHL risk within ten years of blood draw, a novel observation that requires confirmation in other populations.

We observed significant positive associations between CXCL13 and risk of all NHL, B-NHL, and FL, as well as borderline associations with DLBCL and other B-NHL, more than ten years after blood draw, suggesting an early role for an immune environment characterized by B-cell stimulation and aberrant B-cell trafficking. Consistent with this interpretation, a recent, large-scale genome-wide association study of FL identified CXCR5, which is the receptor for CXCL13, as a potential FL susceptibility locus. ${ }^{43}$ Further, genetic variation in CXCR5 and CXCL13 was associated with serum CXCL13 levels in a study of AIDS-NHL, and elevated serum CXCL13 levels were observed in AIDS-NHL cases more than three years prior to diagnosis. ${ }^{2}$ In contrast, elevated sCD30 levels were more strongly associated with increased risk of all NHL, BNHL and FL within ten years of blood draw, with a particularly strong association with FL within five years of blood draw. These findings suggest sCD30 may be capturing a more proximal pre-diagnosis increase in immune activation.

When assessed with multivariable PLR models rather than the main unconditional logistic regression analysis, the associations between immune markers and NHL end points did not change substantially, whether for aggregated end points or the individual B-NHL end points. The minor discrepancies suggested somewhat improved precision in the PLR models, which yielded slightly narrower confidence intervals and slightly stronger $P$-values for heterogeneity by follow-up period for a few of the comparisons. None of the discrepant findings would suggest a different interpretation of the time- or subtype-specific model findings, however, and thus we retained the unconditional logistic regression models as our primary analysis for methodological consistency across the full series of analyses we conducted.

In the analyses with restricted cubic splines, we observed evidence of significant non-linearity for associations of CXCL13 and BAFF with aggregated NHL end points. Of note, those end points comprise small numbers of diverse histological subtypes of NHL which may have different etiologies. Thus, we believe that the observed non-linear associations more likely reflect sampling variability and/or an artifact of potentially heterogeneous subtype-specific associations for the subtypes in the end point groups than a true biological effect.

Together, our findings add new insight to previous publications on both AIDS-NHL and HIV-unrelated NHL risk, collectively suggesting that higher levels of immune activation, and in particular heightened B-cell stimulation, may affect B-cell lymphomagenesis. Interestingly, several markers of immune activation appear to be elevated many years prior to NHL diagnosis and thus could help identify populations at higher risk for developing NHL. It is important to note that some reported associations between immune markers and all NHL risk were not replicated in analyses of individual histological subtypes; this may be due in part to subtype-specific sample sizes that limited statistical power. Significant associations between immune markers and risk of all NHL may reflect commonalities in subtype etiologies; however, these findings may also conceal a more specific association with one or more of the less common subtypes, as illustrated by the present findings for BAFF and CLL/SLL.

This analysis of immune markers measured from prospectively collected blood specimens from two large US cohorts with lengthy follow up identified several statistically significant associations with the risk of developing NHL, including associations that remained statistically significant for blood samples collected five or more years prior to diagnosis.

Although our main results were fairly consistent across analytical approaches, slight variations in markers chosen by a priori and secondary analyses emphasize the importance of utilizing diverse panels of immune markers in future studies seeking to characterize conditions conducive to NHL development. Furthermore, our findings suggest that even though an activated immune milieu may contribute to the development of multiple types of NHL, there is evidence of subtle differences in the pathogenesis of individual NHL subtypes, some of which had not been previously reported. Larger pooled studies will be important to more accurately identify homogeneous and heterogeneous biomarkers of risk or early disease by NHL subtype and to elucidate which are more indicative of earlier or later pathogenic changes to the immune environment.

\section{Acknowledgments}

The authors would like to thank the participants in the Nurses' Health Study and Health Professionals Follow-up Study for their ongoing participation in the cohort studies. We thank Laura Burns for assistance with manuscript preparation, and wish to recognize the technical contributions of the Dana Farber/Harvard Cancer Center Specialized Histopathology Core Laboratory. We thank the following state cancer registries for their help: $A L, A Z$, $A R, C A, C O, C T, D E, F L, G A, I D, I L, I N, I A, K Y, L A, M E$, $M D, M A, M I, N E, N H, N J, N Y, N C, N D, O H, O K, O R, P A$, RI, SC, TN, TX,VA, WA, and WY. The authors assume full responsibility for analyses and interpretation of these data.

\section{References}

1. Takagi R, Higashi T, Hashimoto K, et al. B cell chemoattractant CXCL13 is preferentially expressed by human Th17 cell clones. J Immunol. 2008;181(1):186-189

2. Hussain SK, Zhu W, Chang SC, et al. Serum levels of the chemokine CXCL13, genetic variation in CXCL13 and its receptor CXCR5, and HIV-associated non-hodgkin B-cell lymphoma risk. Cancer Epidemiol Biomarkers Prev. 2013;22(2):295-307.

3. Breen EC, Fatahi S, Epeldegui M, Boscardin
WJ, Detels R, Martinez-Maza O. Elevated serum soluble CD30 precedes the development of AIDS-associated non-Hodgkin's B cell lymphoma. Tumour Biol. 2006;27(4):187-194.

4. Breen EC, van der Meijden M, Cumberland W, Kishimoto T, Detels R, Martinez-Maza O. The development of AIDS-associated Burkitt's/small noncleaved cell lymphoma is preceded by elevated serum levels of interleukin 6. Clin Immunol. 1999;92(3): 293-299

5. Vendrame E, Hussain SK, Breen EC, et al.
Serum levels of cytokines and biomarkers for inflammation and immune activation, and HIV-associated non-Hodgkin B-cell lymphoma risk. Cancer Epidemiol Biomarkers Prev. 2014;23(2):343-349.

6. Edlefsen KL, Martinez-Maza O, Madeleine $\mathrm{MM}$, et al. Cytokines in serum in relation to future non-Hodgkin lymphoma risk: evidence for associations by histologic subtype. Int J Cancer. 2014;135(4):913-922.

7. Saberi Hosnijeh F, Krop EJ, Scoccianti C, et al. Plasma cytokines and future risk of nonHodgkin lymphoma (NHL): a case-control 
study nested in the Italian European Prospective Investigation into Cancer and Nutrition. Cancer Epidemiol Biomarkers Prev. 2010;19(6):1577-1584.

8. De Roos AJ, Mirick DK, Edlefsen KL, et al. Markers of B-cell activation in relation to risk of non-Hodgkin lymphoma. Cancer Res. 2012;72(18):4733-4743.

9. Purdue MP, Lan $Q$, Bagni $R$, et al. Prediagnostic serum levels of cytokines and other immune markers and risk of nonhodgkin lymphoma. Cancer Res. 2011;71(14):4898-4907.

10. Purdue MP, Hofmann JN, Kemp TJ, et al. A prospective study of 67 serum immune and inflammation markers and risk of nonHodgkin lymphoma. Blood. 2013; 122(6):951-957.

11. Purdue MP, Lan Q, Kemp TJ, et al. Elevated serum sCD23 and sCD30 up to two decades prior to diagnosis associated with increased risk of non-Hodgkin lymphoma. Leukemia. 2015;29(6):1429-1431.

12. Purdue MP, Lan Q, Martinez-Maza O, et al. A prospective study of serum soluble CD30 concentration and risk of non-Hodgkin lymphoma. Blood. 2009;114(13):27302732 .

13. Vermeulen R, Hosnijeh FS, Portengen L, et al. Circulating soluble CD30 and future risk of lymphoma; evidence from two prospective studies in the general population. Cancer Epidemiol Biomarkers Prev. 2011;20(9):1925-1927.

14. Gu Y, Shore RE, Arslan AA, et al. Circulating cytokines and risk of $\mathrm{B}$-cell nonHodgkin lymphoma: a prospective study. Cancer Causes Control. 2010;21(8):13231333.

15. Colditz GA, Hankinson SE. The Nurses' Health Study: lifestyle and health among women. Nat Rev Cancer. 2005;5(5):388396.

16. Hankinson SE, Willett WC, Manson JE, et al. Alcohol, height, and adiposity in relation to estrogen and prolactin levels in postmenopausal women. J Natl Cancer Inst. 1995;87(17):1297-1302.

17. Rich-Edwards JW, Corsano KA, Stampfer MJ. Test of the National Death Index and Equifax Nationwide Death Search. Am J Epidemiol. 1994;140(11):1016-1019.

18. Stampfer MJ, Willett WC, Speizer FE, et al. Test of the National Death Index. Am J Epidemiol. 1984;119(5):837-839.

19. Bertrand KA, Giovannucci E, Zhang SM, Laden F, Rosner B, Birmann BM. A prospective analysis of body size during childhood, adolescence, and adulthood and risk of non-Hodgkin lymphoma. Cancer Prev Res (Phila). 2013;6(8):864-873

20. Swerdlow SH, Campo E, Harris NL, et al. WHO Classification of Tumours of Haematopoietic and Lymphoid Tissues.
Lyon, France: IARC Press, 2008.

21. Jaffe ES, Harris NL, Stein H, Vardiman JW Pathology and Genetics of Tumours of Haematopoietic and Lymphoid Tissues. WHO/IARC Classification of Tumours, 3rd Edition, Volume 3. Lyon: International Agency for Research on Cancer, 2001.

22. Morton LM, Turner JJ, Cerhan JR, et al. Proposed classification of lymphoid neoplasms for epidemiologic research from the Pathology Working Group of the International Lymphoma Epidemiology Consortium (InterLymph). Blood. 2007;110(2):695-708.

23. Turner JJ, Morton LM, Linet MS, et al. InterLymph hierarchical classification of lymphoid neoplasms for epidemiologic research based on the WHO classification (2008): update and future directions. Blood. 2010;116(20):e90-98.

24. Rosner B, Cook N, Portman R, Daniels S, Falkner B. Determination of blood pressure percentiles in normal-weight children: some methodological issues. Am J Epidemiol. 2008;167(6):653-666.

25. Rosner B. Percentage Points for a Generalized ESD Many-Outlier Procedure. Technometrics. 1983;25(2):165-172.

26. Wang M, Spiegelman D, Kuchiba A, et al. Statistical methods for studying disease subtype heterogeneity. Stat Med. 2016;35(5):782-800.

27. Ng LG, Sutherland AP, Newton R, et al. B cell-activating factor belonging to the TNF family (BAFF)- $R$ is the principal BAFF receptor facilitating BAFF costimulation of circulating $T$ and $B$ cells. J Immunol. 2004;173(2):807-817.

28. Rihacek M, Bienertova-Vasku J, Valik D, Sterba J, Pilatova K, Zdrazilova-Dubska L. B-Cell Activating Factor as a Cancer Biomarker and Its Implications in CancerRelated Cachexia. Biomed Res Int. 2015;2015:792187.

29. Thompson N, Isenberg DA, Jury EC, Ciurtin C. Exploring BAFF: its expression, receptors and contribution to the immunopathogenesis of Sjogren's syndrome. Rheumatology (Oxford) 2016;55(9):1548-1555.

30. Smedby KE, Hjalgrim $\mathrm{H}$, Askling J, et al. Autoimmune and chronic inflammatory disorders and risk of non-Hodgkin lymphoma by subtype. I Natl Cancer Inst. 2006;98(1):51-60

31. Morton LM, Slager SL, Cerhan JR, et al. Etiologic heterogeneity among nonHodgkin lymphoma subtypes: the InterLymph Non-Hodgkin Lymphoma Subtypes Project. J Natl Cancer Inst Monogr. 2014;2014(48):130-144

32. Ekstrom Smedby K, Vajdic CM, Falster M, et al. Autoimmune disorders and risk of non-Hodgkin lymphoma subtypes: a pooled analysis within the InterLymph Consortium. Blood. 2008;111(8):4029 4038.

33. Herishanu Y, Perez-Galan P, Liu D, et al The lymph node microenvironment promotes B-cell receptor signaling, NF-kappaB activation, and tumor proliferation in chronic lymphocytic leukemia. Blood. 2011;117(2):563-574

34. Endo T, Nishio M, Enzler T, et al. BAFF and APRIL support chronic lymphocytic leukemia B-cell survival through activation of the canonical NF-kappaB pathway. Blood. 2007;109(2):703-710.

35. Planelles L, Castillo-Gutierrez S, Medema JP, Morales-Luque A, Merle-Beral H, Hahne M. APRIL but not BLyS serum levels are increased in chronic lymphocytic leukemia: prognostic relevance of APRIL for survival. Haematologica. 2007;92(9):1284-1285.

36. Bojarska-Junak A, Hus I, Chocholska S, et al. BAFF and APRIL expression in B-cell chronic lymphocytic leukemia: correlation with biological and clinical features. Leuk Res. 2009;33(10):1319-1327.

37. Haiat S, Billard C, Quiney C, AjchenbaumCymbalista F, Kolb JP. Role of BAFF and APRIL in human B-cell chronic lymphocytic leukaemia. Immunology. 2006; 118(3):281-292.

38. Strati P, Shanafelt TD. Monoclonal B-cell lymphocytosis and early-stage chronic lymphocytic leukemia: diagnosis, natura history, and risk stratification. Blood. 2015; 126(4):454-462.

39. Goto $\mathrm{H}$, Tsurumi $\mathrm{H}$, Takemura $\mathrm{M}$, et al Serum-soluble interleukin-2 receptor (sIL2R) level determines clinical outcome in patients with aggressive non-Hodgkin's lymphoma: in combination with the International Prognostic Index. J Cancer Res Clin Oncol. 2005;131(2):73-79.

40. Mir MA, Maurer MJ, Ziesmer SC, et al Elevated serum levels of IL-2R, IL-1RA, and CXCL9 are associated with a poor prognosis in follicular lymphoma. Blood. 2015;125(6):992-998.

41. Goto N, Tsurumi H, Goto H, et al. Serum soluble interleukin-2 receptor (sIL-2R) level is associated with the outcome of patients with diffuse large B cell lymphoma treated with R-CHOP regimens. Ann Hematol. 2012;91(5):705-714.

42. Birmann BM, Breen EC, Stuver $S$, et al. Population differences in immune marke profiles associated with human T-lymphotropic virus type I infection in Japan and Jamaica. Int J Cancer. 2009;124(3):614621

43. Skibola CF, Berndt SI, Vijai J, et al. Genomewide association study identifies five susceptibility loci for follicular lymphoma outside the HLA region. Am J Hum Genet. 2014;95(4):462-471. 